\title{
23.1: Invited Paper: Models and Experiments of Mechanical Integrity for Flexible Displays
}

\author{
Yves Leterrier, Albert Pinyol, Pierre Dumont ${ }^{1}$, Damien Gilliéron, \\ Vinodh Mewani, Jan-Anders Månson \\ Laboratoire de Technologie des Composites et Polymères (LTC), Ecole Polytechnique Fédérale \\ de Lausanne (EPFL), CH 1015 Lausanne, Switzerland
}

(1) present address: Laboratoire de Génie des Procédés Papetiers (LGP2), Ecole Française de Papeterie et des Industries Graphiques (EFPG), BP 65, 461 rue de la papeterie, 38402 Saint-Martin-d'Hères, France

\author{
Janis Andersons \\ Institute of Polymer Mechanics, University of Latvia, Riga LV 1006, Latvia \\ Piet Bouten \\ Philips Research Laboratories, Photonic Materials and Devices, High Tech Campus 4, 5656 AE \\ Eindhoven, The Netherlands \\ Peter Timmermans \\ Philips Applied Technologies, System in Package, High Tech Campus 7, 5656 AE Eindhoven, \\ The Netherlands
}

\begin{abstract}
Flexible displays present a challenging problem in terms of mechanical integrity, a result of the considerable hygro-thermomechanical contrast between the inorganic, brittle device layers and the compliant polymer substrates. This paper reviews the main approaches to study and identify the key factors, which control the mechanical stability of this class of displays. Focus is put on the analyses of residual stress and damage under tensile loading. Novel electro-mechanical methods are used for accurate insight into critical phenomena. An important result is that the thickness and stiffness of the substrate control the critical strain for failure of the device layers.
\end{abstract}

\section{Introduction}

Flexible displays are complex multi-material structures in the form of layered stacks including a polymer or metal substrate, barrier and encapsulation layers, and thin film transistor devices. The integrity of the layer stack and related critical radius of curvature of the display is controlled by the cohesive and adhesive properties and residual stress state of the multi-material assembly. Analysis of the damage resulting from excessive loading during processing and operational life is greatly complicated due to the nanometric dimension of the thin film structures. A further challenge comes from the high mechanical contrast between layers, which may invalidate standard models used to describe the evolution of damage in thin layers. Novel methods and models are therefore needed for accurate measurement of the layer properties and analysis of critical damage events. This information is vital to calibrate numerical tools and enable reliable simulations of display distortion and failure. The present paper presents methods and ongoing developments for the analysis of tensile damage in flexible displays devices, with focus on the deformation of finite compliant substrates.

\section{Hygro-thermo-mechanical properties and residual stresses}

The analysis of display integrity requires accurate input in terms of the properties of the materials constituents (e.g. Young's modulus, coefficients of thermal and of hygroscopic expansion).

\subsection{Elastic properties}

Nano-indentation [1] is well suited to measure the mechanical properties of bulk materials and coatings on hard substrates but not in case of stiff coatings on soft, viscoelastic polymer substrates [2,3]. This is illustrated with the case of indium-doped tin oxide (ITO) coatings on glass and on polyethylene terephthalate (PET; Figure 1). The analysis of the data using the Doerner and Nix model [4] leads to a coating modulus equal to $116 \pm 10 \mathrm{GPa}$ and $22 \pm 3 \mathrm{GPa}$, respectively. Since the two ITO coatings had similar electrical properties, it was inferred that their microstructure, hence their mechanical properties should also be comparable, and not differ by a factor of 5 .
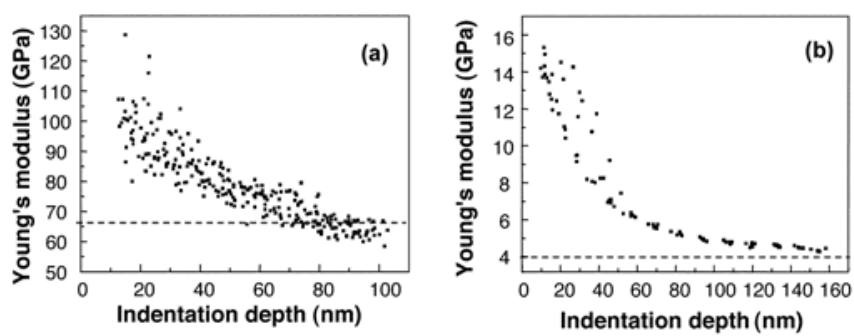

Figure 1. Young's modulus vs indentation depth in case of a $120 \mathrm{~nm}$ thick ITO coating on glass (a) and a $130 \mathrm{~nm}$ thick ITO coating on PET (b). The dotted line represents the substrate modulus

An alternative method is based on the application of classical laminate theory to tensile tests data, using thin polymer substrates. An example is depicted in Figure 2 in case of ITO coatings on 12 
$\mu \mathrm{m}$ thick Arylite ${ }^{\mathrm{TM}}$ films. The analysis of the data leads to a Young's modulus of the ITO coating equal to $119 \pm 5 \mathrm{GPa}$. This value is equal within experimental scatter to the value obtained by nanoindentation on a stiff substrate.

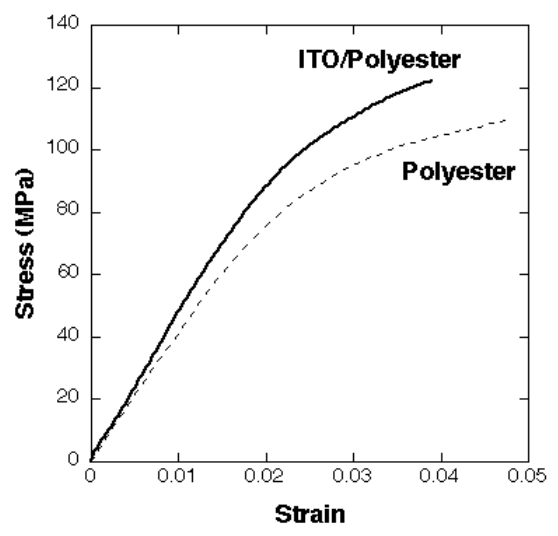

Figure 2. Tensile behavior of a $12 \mu \mathrm{m}$ thick polyester film, with and without a $100 \mathrm{~nm}$ thick ITO coating

\subsection{Residual stresses and hygrothermal properties}

In the case of thin inorganic films deposited from a vapor phase onto polymer or metal substrates, residual stresses comprise intrinsic and thermal contributions, and, in case of polymer substrates, a hygroscopic contribution [5, 6], as depicted in Figure 3. An approach to identify the stress contributions is to monitor the curvature of the multilayer film subjected to iso-hygric thermal ramps, and isothermal relative humidity jumps. The resulting curvature changes are subsequently analyzed using hygro-thermo-mechanical modeling framework [6]. This approach moreover enables to obtain the coefficients of thermal and hygroscopic expansion (CTE and CHE, respectively) of thin coatings.

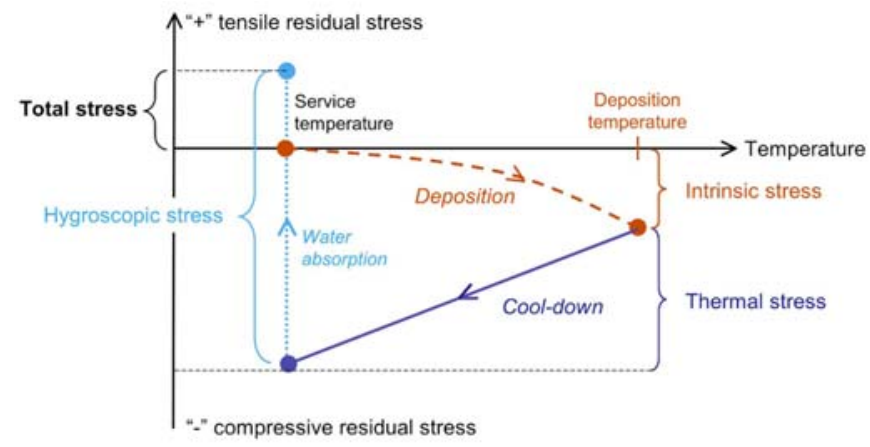

Figure 3. Residual stress dynamics during processing of thin films.

\section{Failure under tensile stress}

Tensile stresses build up in flexible display structures under tensile loads, as also upon heating (thermal stress) and bending. Excessive levels of tensile stresses relax through micro-cracking and, depending on adhesion quality, also through interfacial delamination. The fragmentation test (a tensile test in situ in a microscope) was found to be well suited for accurate determination of damage processes. The main advantage of the method is the absence of third-body interactions and associated complicated corrections factors. It enables to derive cohesive and adhesive properties of brittle coatings [7,8]. Figure 4 represents fragmentation data for $50-800 \mathrm{~nm}$ thick $\mathrm{SiN}_{\mathrm{x}}$ coatings on two different $125 \mu \mathrm{m}$ thick polyimide substrates.
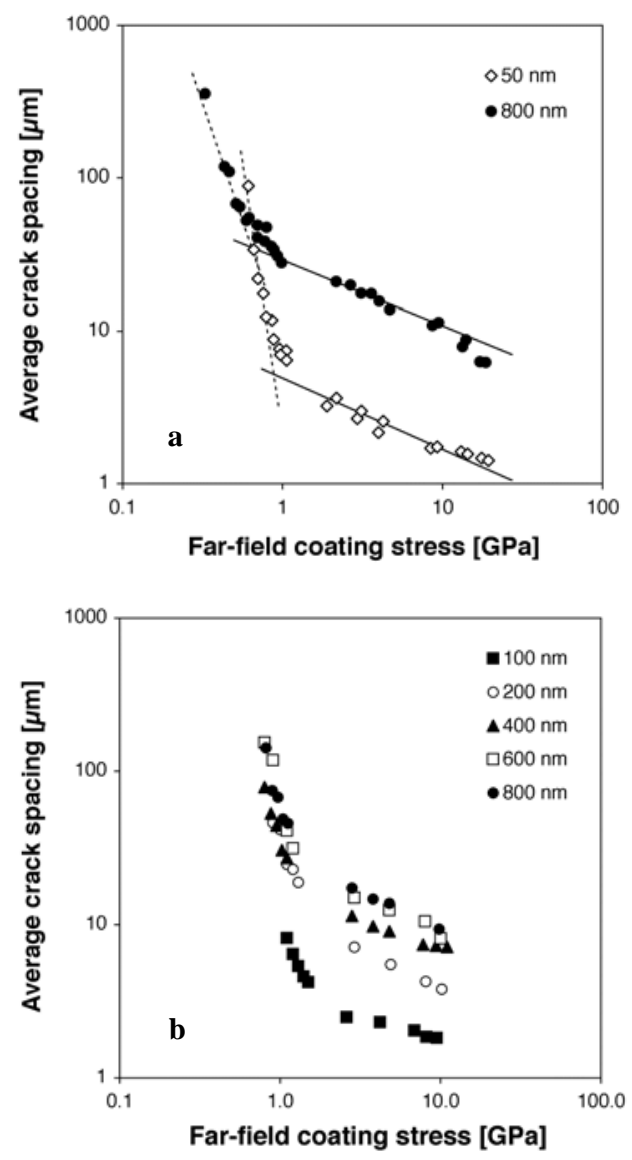

Figure 4. Average crack spacing vs coating tensile stress for $\mathrm{SiN}_{\mathrm{x}}$ coatings on polyimide (Kapton: a; Upilex: b), with model shown in case of Kapton (see text for details).

Two typical stages of fragmentation are clearly discerned. The initial fragmentation stage starts with critical failure stress (associated with critical radius of curvature in case of bending load) and is characterized by rapid multiplication of cracks with the increase of load. This is due to the typical crack spacing being much larger than the stress transfer length. Therefore, the rate of crack generation is governed solely by the coating strength distribution and coating stress. This feature is used to identify the parameters of Weibull strength distribution (that corresponds to power-law relation between crack spacing and coating stress shown by dashed lines in Figure 4a). The advanced fragmentation stage sets in with the onset of crack interaction. The cracking rate and the spacing distribution in this stage depend mainly on stress transfer efficiency between substrate and coating. For shear lag model of stress transfer, explicit relation of crack spacing as a function of stress, coating strength distribution parameters, and stress transfer length is derived in [8]. The relation (solid lines in Figure 4a) allows quantitative characterization of interface mechanical efficiency via effective stress transfer length, determined from the advanced fragmentation data. 


\section{Electro-mechanical methods}

The above fragmentation method is accurate but time consuming. Two novel methods, electro-fragmentation and electro-fatigue were developed for automatic measurement of critical failure strain and damage under fatigue loading [9]. The key concept is to explore the development of damage with simultaneous observation in-situ in a microscope and monitoring of the electrical properties. Both methods rely on an ultrathin graphite layer used as a conductive probe in case of testing dielectric coatings such as $\mathrm{SiO}_{2}$ and $\mathrm{SiN}_{\mathrm{x}}$ passivation and diffusion barriers. A careful optimization of the conductive probe layer was carried out to avoid artifacts resulting for instance from a change of the residual stress state of the investigated coating.

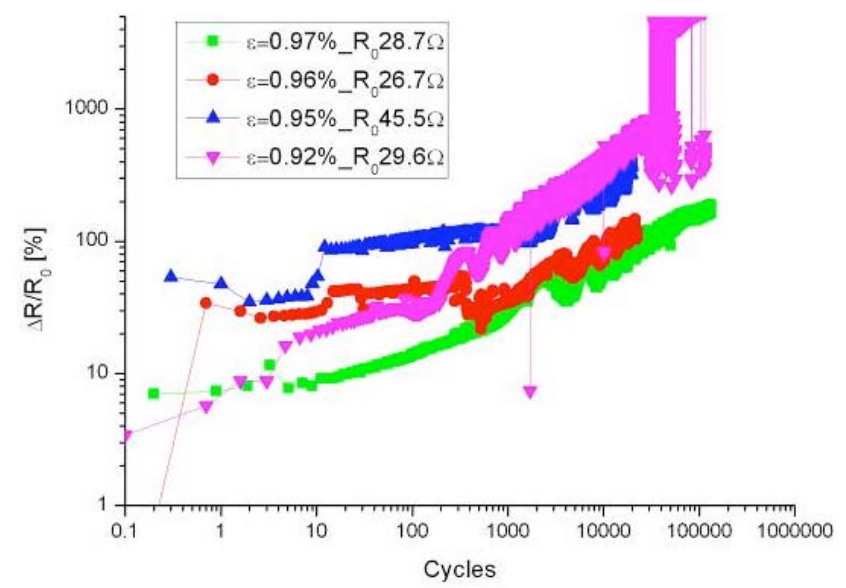

Figure 5. Normalized electrical resistance increase of a $100 \mathrm{~nm}$ thick transparent conductive oxide film on PET under cyclic loading between 0 and maximum strain $(0.92-0.97 \%)$.

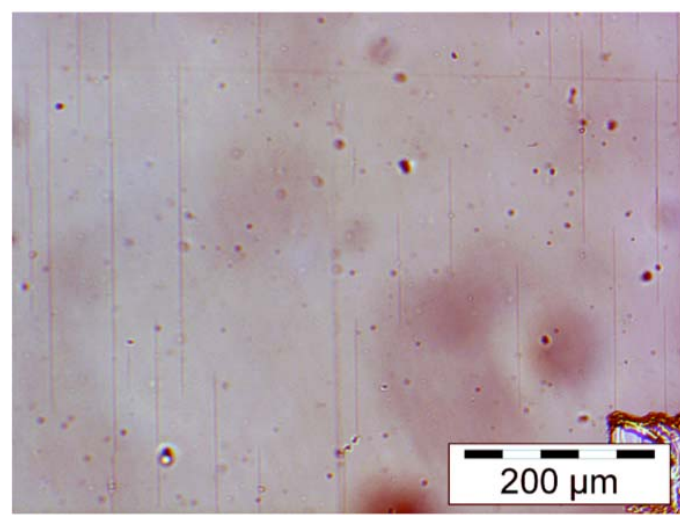

Figure 6. Damage state (tensile cracks of finite length) in a $100 \mathrm{~nm}$ thick transparent conductive oxide film on PET after 1 '700 cycles to $0.97 \%$ tensile strain (the loading was parallel to the fiducial mark).

Figure 5 depicts the change of electrical resistance during cyclic loading at strain levels close to $80 \%$ of the crack onset strain, for a transparent conducting oxide film on PET. Detailed analysis of the onset of tensile damage reveals the existence of very long stable cracks in the brittle coating as shown in Figure 6, and a progressive transition towards unstable failure. The consequence of this process is that sub-critical cracks may grow under fatigue loading until catastrophic failure, the details of which being essential for proper theoretical analysis. These methods enable to simulate a broad range of thermo-mechanical scenarios, and are therefore well suited for accurate insight into critical processes that control the lifetime stability of flexible electronic devices.

\section{Analysis of mechanical contrast influence on failure strain}

The influence of the mechanical properties of the substrate on the cracking of thin layers has been studied theoretically using fracture mechanics concepts by several authors (e.g., [10]). In the case of compliant polymer substrates, cracking is predicted to occur at a lower strain compared to stiff substrates. Such analysis was carried out for $\mathrm{SiO}_{2}$ films used as dielectric passivation layers in 4-level TFT structures for $152 \mu \mathrm{m}$ thick steel and $8 \mu \mathrm{m}$ thick polyimide (PI) substrates. The $\mathrm{SiO}_{2}$ film thickness was $1 \mu \mathrm{m}$ on steel, and $1.28 \mu \mathrm{m}$ on PI. Cracks initiated at $1.15 \%$ strain and $0.24 \%$ strain on steel and PI, respectively. The latter value is similar to data reported in literature [11]. Such a large difference in critical strain for the $\mathrm{SiO}_{2}$ layer on the two types of substrates was analyzed starting from fracture mechanics concepts.
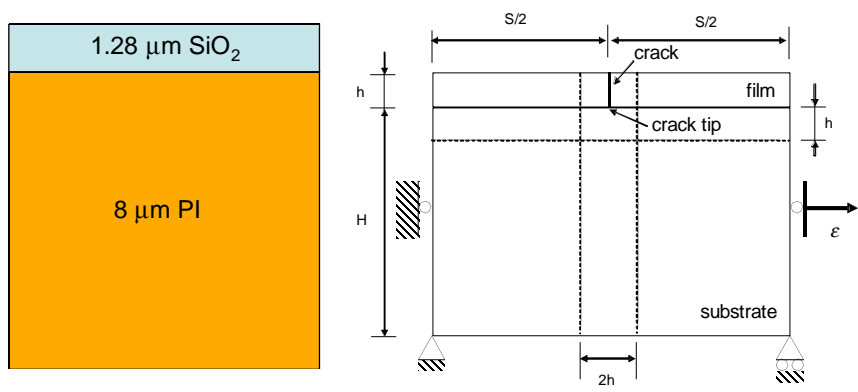

Figure 7. Geometry used to model the behavior of a crack in a brittle layer on a finite compliant substrate.

First, the energy release rate of a crack in the $\mathrm{SiO}_{2}$ layer on steel and PI was calculated assuming semi-infinite substrates [10]. Following this approach, the failure strain of the $\mathrm{SiO}_{2}$ layer on the steel substrate was found to be a factor of 2 higher compared to the PI substrate. The behavior of the $\mathrm{SiO}_{2}$ layer on a finite thickness PI substrate was analyzed using the Finite Element Method (FEM), using the model geometry shown in Figure 7. From the FEM calculations the average opening of the crack over the film thickness was taken and the method outlined in [12] was used to calculate the energy release rate. Figure 8 gives a typical result for a finite element calculation at $S / h=100$, in case of the thin PI film. The contour bands indicate the deformation in $y-$ direction, scaled by a factor of 50 . In order to arrive at the steady state value of the energy release rate a crack spacing $\mathrm{S}$ of 500 times the film thickness $h$ was considered. Figure 9 depicts calculated values of the elastic mismatch function $g$ [10] (the higher the $g$, the lower the strain to failure) vs. layer-to-substrate stiffness times thickness ratio, representing the load carrying contribution (or tensile stiffness) of the film with respect to the substrate. A nearly linear relation is evident, which permits the estimation of $g$, hence the influence of geometry and elastic contrast on cracking strain for small geometry variations. 


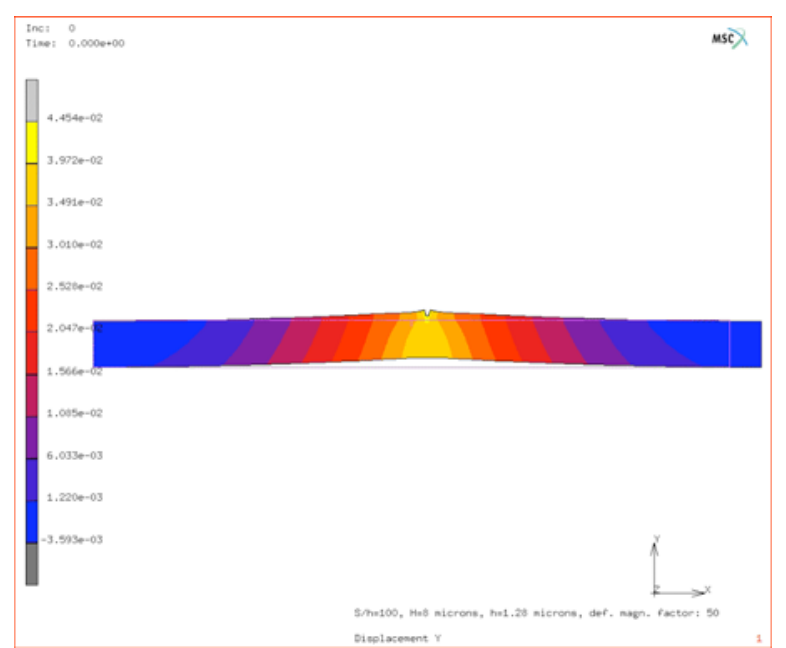

Figure 8. Finite element simulation of the deformation of a $1280 \mathrm{~nm}$ thick $\mathrm{SiO}_{2}$ on a $8 \mu \mathrm{m}$ thick PI substrate at $\mathrm{S} / \mathrm{h}=100$.

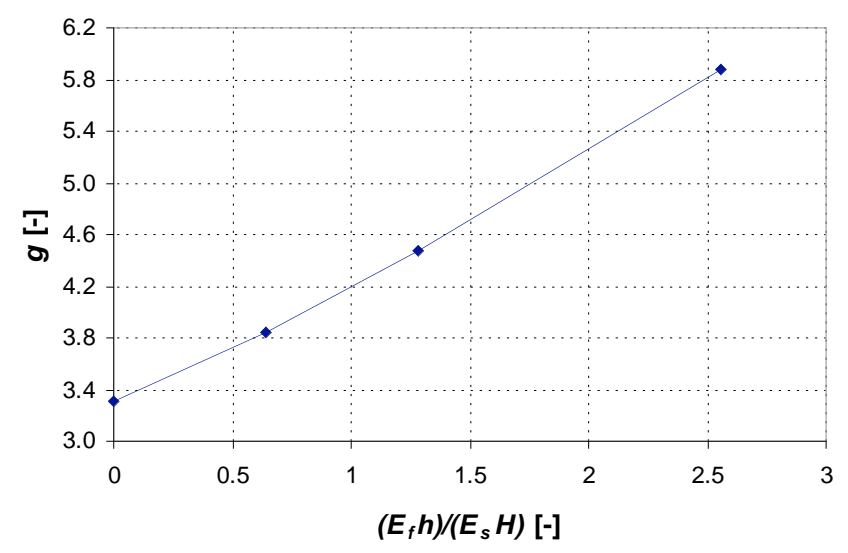

Figure 9. Elastic mismatch function $\mathrm{g}$ as a function of layer/substrate geometry and stiffness.

The measured difference in failure strain of the brittle layer on steel or thin polymer substrates is well understood, when the contributions due to thermal mismatch, elastic mismatch, the finite substrate thickness and the (small) difference in the applied brittle layer thickness are properly taken into account.

\section{Conclusions}

Reliable modeling of the failure behavior of flexible displays based on multilayer stacks requires accurate determination of the mechanical and hygrothermal properties of material constituents, and corresponding residual stress state. The residual stress state comprises intrinsic, thermal, and hygroscopic contributions, that can be identified through modeling of selected hygrothermal loading paths. The fragmentation test, and its extension with simultaneous electrical measurements are valuable tools in the analysis of tensile damage evolution. This method enables to derive the cohesive and adhesive properties of the layers. The critical failure strain of $\mathrm{SiO}_{2}$ passivation layers was found to be four times higher in case of a stiff and thick steel substrate compared to a compliant and thin polymer substrate. Such a large difference was analyzed using numerical methods and found to result mainly from differences in layer/substrate geometry and elastic contrast, combined with differences in thermal strain.

\section{Acknowledgments}

The EC is acknowledged for funding of this work under contract IST-2004-4354 (FlexiDis), and the Fraunhofer-IST is also acknowledged for the nano-indentation tests. The companies Applied Materials and Unaxis Balzers are acknowledged for supplying some of the film samples.

\section{References}

[1] W.C. Oliver, G.M. Pharr, Measurement of hardness and elastic modulus by instrumented indentation: Advances in understanding and refinements to methodology, J. Mater. Res. 19/1 (2004) 3.

[2] R. Saha, W.D. Nix, Effects of the substrate on the determination of thin film mechanical properties by nanoindentation, Acta Mater. 50/1 (2002) 23.

[3] T. Staedler, K. Schiffmann, Correlation of nanomechanical and nanotribological behavior of thin DLC coatings on different substrates, Surf. Sci. 482 (2001) 1125.

[4] M.F. Doerner, W.D. Nix, A method for interpreting the data from depth-sensing indentation instruments, J. Mater. Res. 1 (1986) 601.

[5] S. Wagner, H. Gleskova, I.-C. Cheng, J.C. Sturm, Z. Suo, in Flexible Flat Panel Displays, Wiley, New York, 2005.

[6] P. Dumont, G. Tornare, Y. Leterrier, J.-A.E. Månson, Intrinsic, thermal and hygroscopic residual stresses in thin gas-barrier films on polymer substrates, Thin Solid Films 515/19 (2007) 7437.

[7] Y. Leterrier, Durability of Nanosized Gas Barrier Coatings on Polymers, Prog. Mater. Sci. 48 (2003) 1.

[8] J. Andersons, Y. Leterrier, G. Tornare, P. Dumont, J.-A.E. Månson, Evaluation of Interfacial Stress Transfer Efficiency by Coating Fragmentation Test, Mech. Mater. 39 (2007) 834.

[9] A. Pinyol, B. Meylan, D. Gilliéron, A. Mottet, V. Mewani, Y. Leterrier, J.-A.E. Månson, Electro-Fragmentation Analysis of Dielectric Thin Films on Flexible Polymer Substrates, Proc. MRS Fall Meeting, Boston, MA, November 26-30, 2007.

[10] J.L. Beuth, Cracking of Thin Bonded Films in Residual Tension, Int. J. Solids Struct. 29/13 (1992) 1657.

[11] H. Gleskova, I.C. Cheng, S. Wagner, J.C. Sturm, Z. Suo, Mechanics of thin-film transistors and solar cells on flexible substrates, Solar Energy 80/6 (2006) 687.

[12] R. Huang, J.H. Prévost, Z.Y. Huang, Z. Suo, Channelcracking of thin films with the extended finite element method, Eng. Fract. Mech. 70 (2003) 2513. 